\author{
S.D. Braun \\ G. Schmidt \\ K. Krumbholz \\ T. Störk \\ R. Gareis
}

Eingegangen: 2. Februar 2004

Akzeptiert: 10. März 2004

Herrn Prof. Dr. med. Hans Hochrein zum 75. Geburtstag gewidmet

S.D. Braun

Klinik für Kardiologie

Medizinische Klinik und Poliklinik II

Ärztliche Direktorin:

Prof. Dr. med. R. H. Strasser

Technische Universität Dresden

Dr. med. R. Gareis ( $\bullet$. S.D. Braun

G. Schmidt · T. Störk

Innere Klinik II

Kardiologie, Angiologie

und Internistische Intensivmedizin

Chefarzt: Prof. Dr. med. T. Störk

Karl-Olga-Krankenhaus

Akademisches Lehrkrankenhaus

der Universität Ulm

Hackstraße 61

70190 Stuttgart, Germany

Tel.: $0711 / 2639-2400$

Fax: $0711 / 2639-2430$

E-Mail:

Ragnar.Gareis@Karl-Olga-Krankenhaus.de

K. Krumbholz

Zentrale Röntgenabteilung

Chefarzt: Dr. med. K. Krumbholz

Karl-Olga-Krankenhaus Stuttgart

Akademisches Lehrkrankenhaus

der Universität Ulm

\title{
Nichtinvasives Beatmungsregime und liberales Volumenmanagement bei schwerem ARDS mit septischer Genese - eine Kasuistik
}

Summary Early stages of ARDS are characterized by pulmonary edema caused by a inflammatory mediated leakage of the capillaryalveolar membranes. Therefore restrictive fluid management is commonly recommended. The main reason for ARDS, however, is sepsis. In sepsis, fluid restriction may be harmful because this can attenuate cardiac output while hyperdynamic circulation is needed to maintain aerobic metabolism of the non-pulmonary organs. So if ARDS is caused by sepsis we face a therapeutic dilemma, on the one hand, to reduce circulatory fluid to improve ventilation and, on the other hand to raise circulatory fluid to optimize hemodynamics.

Another important treatment approach of ARDS is early mechanical ventilation with intubation. Mechanical ventilation however can induce further progression of lung injury. Besides using low-volume pressure-limited ventilation with permissive hypercapnia, non-invasive ventilation has recently been proposed as an efficient alternative to conventional mechanical ventilation in the treatment of ARDS.

We present a case report of ARDS caused by urosepsis in a young patient. The positive course with fast recovery is associated, in our opinion, to the causal relationship of a non-invasive ventila- tion regiment and liberal fluid management.

Key words ARDS ventilator induced lung injury VILI - non-invasive ventilation sepsis - hemodynamics

Zusammenfassung In der Frühphase des ARDS entwickelt sich als Folge einer Permeabilitätsstörung der alveolo-kapillären Membran ein Lungenödem trotz normalen pumonalkapillären und kolloidosmotischen Druckes. Daher ist eine Säule in der Therapie des ARDS ein äußerst restriktives Volumenmanagement. Häufigste Ursache des ARDS jedoch ist eine Sepsis. Die Sepsis ist typischerweise gekennzeichnet durch eine Sauerstoffverwertungsstörung auf zellulärer Ebene. Zur Aufrechterhatlung eines aeroben Stoffwechsels der nicht-pulmonalen Organe ist dann ein erheblich gesteigertes Herzzeitvolumen mit deutlichem Abfall des systemischen vaskulären $\mathrm{Wi}$ derstandes (SVR) erforderlich. In dieser Situation ist aber ein Volumenentzug mit der Folge des Abfalles des Herzzeitvolumens äußerst problematisch. Sollte das ARDS also durch eine Sepsis ausgelöst sein, so besteht ein therapeutisches Dilemma, einerseits Flüssigkeit entziehen zu müssen, um den pulmonalen Gasaustausch $\mathrm{zu}$ verbessern, andererseits aber 
Flüssigkeit zuführen zu müssen, um die Organperfusion im Rahmen der Sepsis zu optimieren.

Als weiterer Baustein der Therapie des ARDS gilt eine frühzeitige, invasiv-maschinelle Beatmung. Zunehmend werden jedoch auch beim ARDS nicht-invasive Beatmungsformen diskutiert, da sich gezeigt hat, dass die invasive, maschinelle Überdruckbeatmung über einen Tubus die Lunge zusätzlich schädigen können.

Wir berichten über den Fall eines schweren ARDS im Rahmen einer Urosepsis bei einer jungen, bisher gesunden Patientin. Den erfreulich positiven Verlauf mit relativ rascher Restitution sehen wir im Zusammenhang mit einem nichtinvasiven Beatmungsregime und liberaler Volumenbilanzierung.

Schlüsselwörter ARDS Nicht-invasive Beatmung Ventilator induzierte Lungenschädigung - Sepsis Hämodynamik

\section{Einleitung}

ARDS steht für Adult Respiratory Distress Syndrome und bezeichnet ein akutes Organversagen der Lunge. Erstmals wurde es von Ashbaugh schon 1967 beschrieben [1]. Die noch heute aktuellen Kriterien zur Diagnose eines ARDS wurden von der Amerikanisch-Europäischen Konsensus-Konferenz 1994 festgelegt ([2] Tab. 1). Pathophysiologisch liegt dem ARDS eine Mediator-induzierte Schädigung der alveolo-kapillären Membran mit einer Permeabilitätsstörung der Lungenkapillaren zugrunde. Aufgrund der pathologisch gesteigerten Kapillardurchlässigkeit bildet sich initial ein Lungenödem trotz eines normalen pulmonalkapillären und kolloidosmotischen Drucks. Nach der exsudativen Frühphase folgt im Intermediärstadium eine alveoläre Verdichtung mit Fibrinablagerungen, Untergang von Typ-II-Pneumocyten, dadurch verminderter Surfactant Produktion [3], sowie eine Mikro- und Makrothrombosierung der pulmonalen Strombahn. Durch die entstehenden Mikroatelektasen bilden sich intrapulmonale Shunts mit zwar perfundierten, aber nicht ventilierten Lungenarealen und einer konsekutiven Sauerstoff-refraktären Hypoxämie. Als Ausdruck der vermehrten Shuntperfusion findet sich eine erhöhte alveolo-arterielle Differenz des Sauerstoffpartialdrucks. Typischerweise besteht ein direktes Nebeneinander von funktionell normalen und schwerst veränderten Arealen. Im Spätstadium erfolgt ein fibroproliferativer Umbau des Lungengerüstes mit der Folge eines irreversiblen respiratorischen Versagens [4]. Die frühere Bezeichnung Schocklunge bringt den in der Regel dramatischen klinischen Verlauf mit akutem respiratorischen Versagen gut zum Ausdruck. Noch immer handelt es sich um ein gefürchtetes Krankheitsbild in der Intensivmedizin mit Mortalitätsraten bis zu 80\%. Häufigste Ursache des ARDS ist die Sepsis, gefolgt von der Pneumonie und Schockzuständen $[5,6]$.

Aufgrund der hohen Mortalität und der irreversiblen strukturellen Schädigung in der Spätphase ist die frühzeitige Erkennung des Krankheitsbildes wichtig [7]. Abgesehen von der Klinik und den respiratorischen Parametern beruht die Diagnostik entscheidend
Tab. 1 Empfohlene Kritierien zur Diagnose eines ARDS [2]

\begin{tabular}{l}
\hline Akuter Beginn \\
\hline $\mathrm{paO}_{2} / \mathrm{FiO}_{2}<200 \mathrm{mmHg}$ (unabhängig vom PEEP) \\
Bilaterale Infiltrationen im Röntgen-Thorax \\
$\mathrm{PcP}<18 \mathrm{mmHg}$ oder kein klinischer Anhalt für links-atriale Hypertonie \\
\hline
\end{tabular}

auf radiologischen Verfahren. Im konventionellen Röntgen-Thorax finden sich beim ARDS bilaterale, diffuse Infiltrate, die sich von einem kardialen Lungenödem nur schwierig differenzieren lassen. Charakteristische, stadienabhängige Veränderungen finden sich jedoch in der Thorax-Computertomographie (CT). Das Frühstadium ist durch eine milchglasartige Eintrübung beider Lungenflügel und betonte interstitielle Septen gekennzeichnet. In der Intermediärphase zeigt die CT diffuse, fleckig konfluierende pulmonale Verdichtungen mit Betonung der abhängigen Lungenpartien. Im Spätstadium findet sich eine grob retikuläre Zeichnungsvermehrung und ein interstitielles Emphysem mit kettenartiger, peribronchovaskulärer Anordnung kleiner Pneumatozelen, welche zum Pneumothorax prädisponieren und als Zeichen einer schlechten Prognose gedeutet werden müssen [8]. Unabhängig vom auslösenden Agens läuft die Mediator vermittelte pathophysiologische Sequenz uniform ab, so dass die Histologie keine Rolle spielt. Als Therapieregime der Wahl gilt zur Zeit die frühzeitige, so genannte "lungenprotektive" maschinelle Beatmung über einen endotrachealen Tubus, mit niedrigen Atemzugvolumina und dadurch bedingt niedrigen Beatmungsspitzendrücken unter Tolerierung erhöhter arterieller $\mathrm{CO}_{2}$-Werte („permissive Hyperkapnie“). Zudem sollte immer auch ein PEEP (positive endexspiratory pressure) eingestellt werden, wobei die Höhe des besten PEEP noch unklar ist [9-12].

Zunehmend werden jedoch auch bei dem ARDS nichtinvasive Beatmungsformen diskutiert, da sich gezeigt hat, dass die invasive, maschinelle Überdruckbeatmung über einen Tubus die Lunge zusätzlich schädigen und den Krankheitsverlauf negativ beeinflussen können $[13,14]$. 
Als ein weiterer Baustein der ARDS-Therapie gilt aufgrund der Permeabilitätsstörung der Lungenkapillaren und der damit verbundenen pulmonalen Flüssigkeitseinlagerungen bei normalen linksatrialen Drücken, eine negative Flüssigkeitsbilanzierung [15]. Bei Sepsis hingegen konnte in einer kürzlich veröffentlichten Studie ein positiver Einfluss auf die Mortaliät durch frühe Anhebung des zentralvenösen Druckes auf hochnormale Werte gezeigt werden [16]. Sollte das ARDS also durch eine Sepsis ausgelöst sein, so besteht ein therapeutisches Dilemma, einerseits Flüssigkeit entziehen zu müssen, um den pulmonalen Gasaustausch zu verbessern [17], andererseits aber Flüssigkeit zuführen zu müssen, um die Organperfusion im Rahmen der Sepsis zu optimieren [18-20]. Einige Autoren favorisieren in derartigen Situationen einen Mittelweg [21, 22].

Wir berichten über den Fall eines schweren ARDS im Rahmen einer Urosepsis mit kompletter Restitution unter einem frühzeitigen, nichtinvasiven Beatmungsregime und liberaler Volumensubstitution.

\section{Kasuistik}

\section{Anamnese, periphere Station}

Die stationäre Aufnahme der 20-jährigen Patientin erfolgte initial auf die Urologische Abteilung wegen seit drei Tagen bestehender Flankenschmerzen rechts, begleitet von Fieber bis $40^{\circ} \mathrm{C}$. Anamnestisch keine weiteren Vorerkrankungen. Aufgrund der klinischen Befunde und laborchemisch erhöhter Entzündungsparameter erfolgte eine Uro-Sonographie. Hier zeigte sich eine Urolithiasis rechts mit Stein im Ureter und dilatiertem Nierenbeckenhohlsystem. Es erfolgte ein retrogrades Pyelogramm und eine transurethrale Ureteroskopie. Da die Extraktion des Steins nicht möglich war wurde der Stein ins rechte Nierenbecken mobilisiert und ein Doppel-J-Katheter eingelegt. Während der Intervention entleerte sich eitrig-trübes Sekret aus dem Nierenbeckenkelchsystem. Am Tag nach erfolgter Intervention kam es zur dramatischen Verschlechterung des klinischen $\mathrm{Zu}$ standes der jungen Patientin, weshalb eine Verlegung auf die Intensivstation erfolgte.

\section{Klinischer Aufnahmebefund, Intensivstation}

20-jährige Patientin, Körpergröße $158 \mathrm{~cm}$, Gewicht $55 \mathrm{~kg}$, wach, allseits orientiert, kritisch kreislaufstabil bei einer Tachycardie von 140/min und hypotensiven Blutdruckwerten von $80 / 60 \mathrm{mmHg}$. Ausgeprägte Tachypnoe, Ruhedyspnoe, keine Orthopnoe, Hustenreiz, kein Auswurf. Hautkolorit blass, keine Cyanose, keine Ödeme. Seitengleiche Atmung, sonorer Klopfschall, verschärftes vesikuläres Atemgeräusch. Herztöne rein, Herzaktion rhythmisch, tachycard. Bauchdecken weich, Darmgeräusche regelrecht, klopfschmerzhaftes rechtes Nierenlager. Orientierend neurologische Untersuchung und peripherer Pulsstatus unauffällig.

\section{Klinisch-chemische Befunde}

Pulsoxymetrisch ermittelte Sauerstoffsättigung $\left(\mathrm{SaO}_{2}\right)$ ohne Sauerstoffgabe $48 \%$. Arterielle Blutgasanalyse unter der Zufuhr von $91 \mathrm{O}_{2}$ /min: $\mathrm{pH} 7,5, \mathrm{PaO}_{2} 33 \mathrm{mmHG}$, $\mathrm{PaCO}_{2} 36 \mathrm{mmHg}$, Standardbikarbonat $29 \mathrm{mmol} / \mathrm{l}$, Basenüberschuss $6,5, \mathrm{SaO}_{2} 71 \%$. Weitere pathologische Laborwerte waren: Leukocytose mit 10900 Leukocyten $/ \mu$, Granulocytose und relative Lymphopenie, $14 \%$ Stabkernige, grenzwertige Thrombopenie mit $136000 / \mu 1$, Koagulopathie bei einer Thromboplastinzeit von 64\%, C-reaktives Protein erhöht auf $314 \mathrm{mg} / \mathrm{l}$, Fibrinspaltprodukte erhöht auf $2000 \mu \mathrm{g} / \mathrm{l}$, Fibrinogen erhöht auf $680 \mathrm{mg} / \mathrm{dl}$. Im Urinstatus massenhaft Leukocyten, dreifach positiver Bakteriennachweis, leichte Proteinurie. In der Urinkultur Wachstum von Escherichia coli in signifikanter Keimzahl. Die übrigen Routinelabor-Parameter waren in der Norm.

\section{Ergänzende Untersuchungen}

Radiologisch zeigte sich im konventionellen RöntgenThorax eine erhebliche, bis in die Lungenperipherie reichende Eintrübung beider Lungen mit geringer Restbelüftung in den Lungenoberfeldern, ein Erguss war nicht abgrenzbar, die Herzgröße war im Normbereich (Abb. 1).

Echokardiographisch war der linke Ventrikel normal groß mit global und regional guter Pumpfunktion. Der rechte Ventrikel war mit $30 \mathrm{~mm}$ vergrößert, wenn auch nicht spitzenbildend. Die Aortenklappe und Mitralklappe zeigten keine Auffälligkeiten. Aufgrund einer Trikuspidalinsuffizienz ersten Grades konnte ein rechtsventrikulär-endsystolischer Druck von $40 \mathrm{mmHg}$ ermittelt werden, einer mäßigen, pulmonalen Hypertonie entsprechend.

Zur differentialdignostischen Abklärung der Lungeninfiltrationen und der Rechtsherzbelastungszeichen, erfolgte die Durchführung einer Thorax-CT. Mit Ausnahme der Lungenoberfelder war die gesamte Lunge komplett mit einem Exsudat im Sinne eines ARDS in der exsudativen Phase ausgefüllt. Rechts war ein schmaler Pleuraerguss nachweisbar (Abb. 3, 5). Nebenbefundlich konnte eine zentrale Lungenembolie ausgeschlossen werden. Abdomensonographisch fand sich das Bild einer Pyelonephritis rechts, 


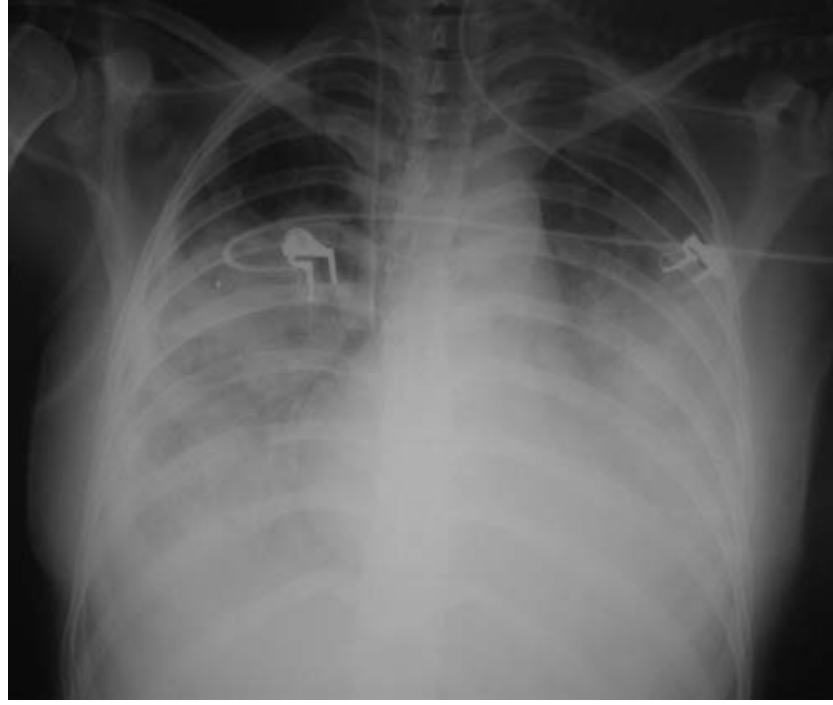

Abb. 1 Röntgen-Thorax der Lunge bei Aufnahme: beidseits milchglasartige Eintrübungen der Lungen

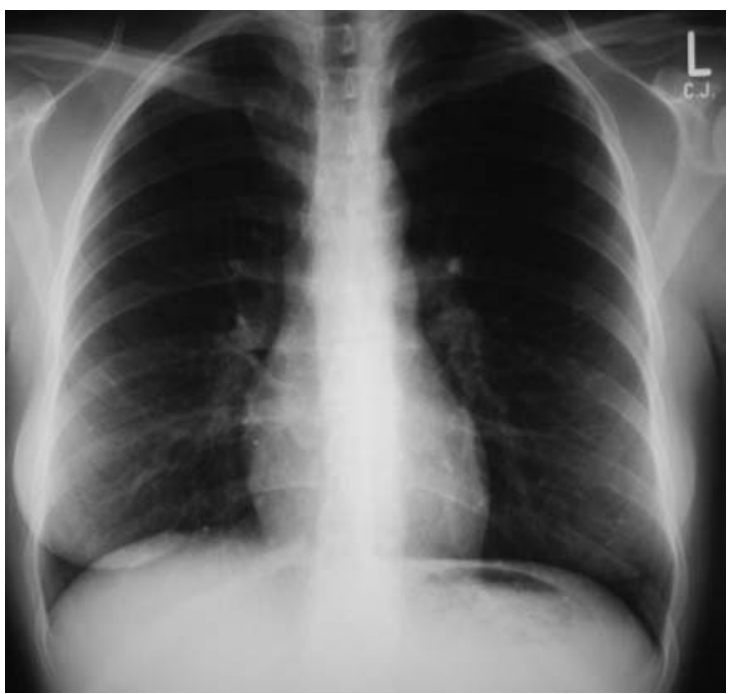

Abb. 2 Röntgen-Thorax-Kontrolle vor Verlegung der Pat. Vollständige Rückbildung der Infiltrate

mit Nierenstein im oberen Nierenbecken, bei liegendem DJ-Katheter.

Elektrokardiographisch fand sich eine Sinustachycardie mit unspezifischen Erregungsrückbildungsstörungen in den Brustwandableitungen.

\section{Diagnose}

ARDS bei Urosepsis mit Multiorganversagen (Lungenveragen, Koagulopathie und Kreislaufinsuffizienz), konsekutive pulmonale Hypertonie.

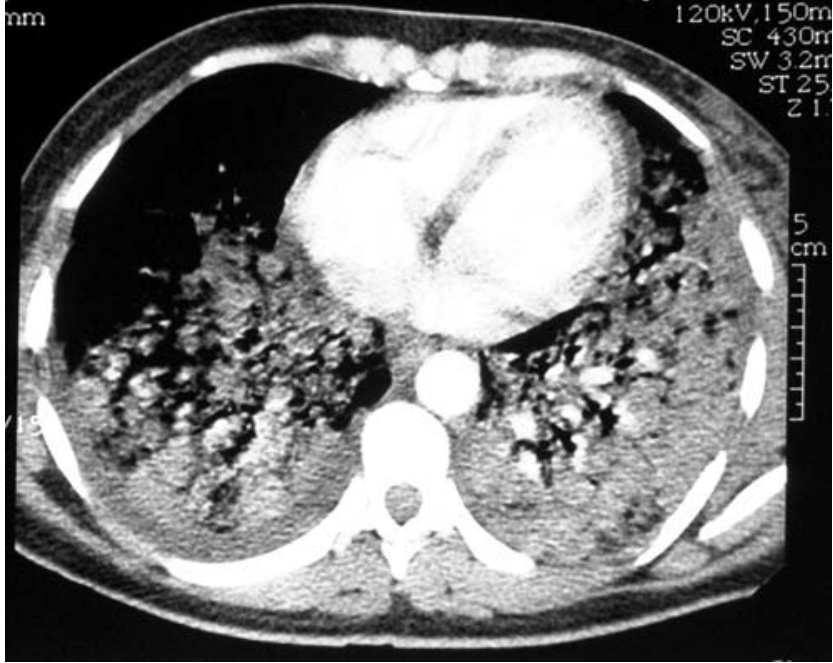

Abb. 3 CT-Thorax kurz nach Aufnahme. Ausgeprägte Infiltrationen bds., dorsobasal betont

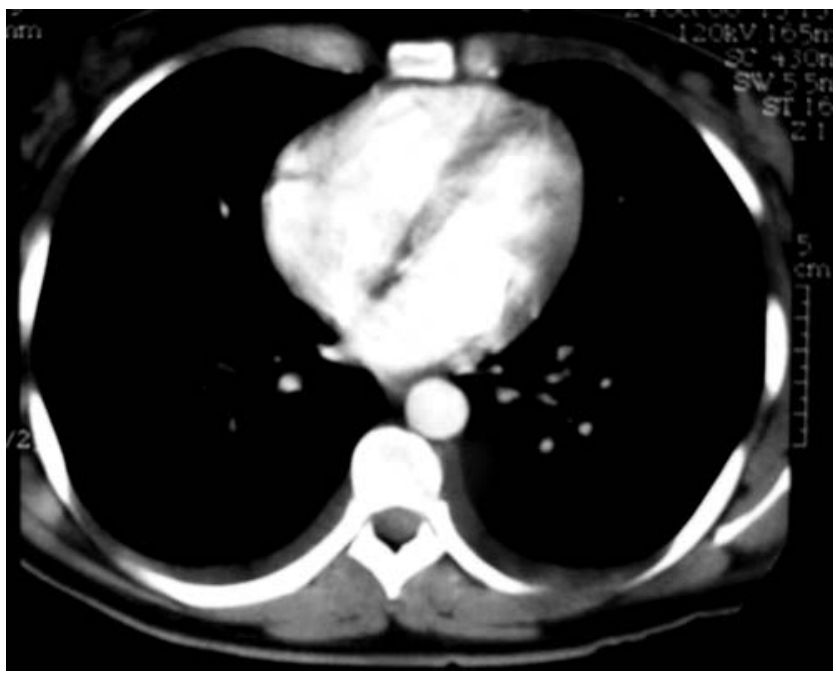

Abb. 4 CT-Thorax-Kontrolle vor Verlegung der Pat. Vollständige Rückbildung der Infiltrate

\section{Therapie und Verlauf}

Aufgrund der erhaltenen Vigilanz und guter Patienten-Compliance entschlossen wir uns trotz ausgeprägter Hypoxämie und entgegen der üblichen Praxis, zur Durchführung einer möglichst schonenden nichtinvasiven Beatmungsstrategie mit CPAP. Hierdurch sollte das Risiko beatmungsbedingter Komplikationen minimiert werden. Aufgrund der Urosepsis mit vorliegendem Multiorganversagen (Koagulopathie, Schocklunge, Kreislaufinsuffizienz mit positivem Schockindex) erfolgte zur Optimierung 


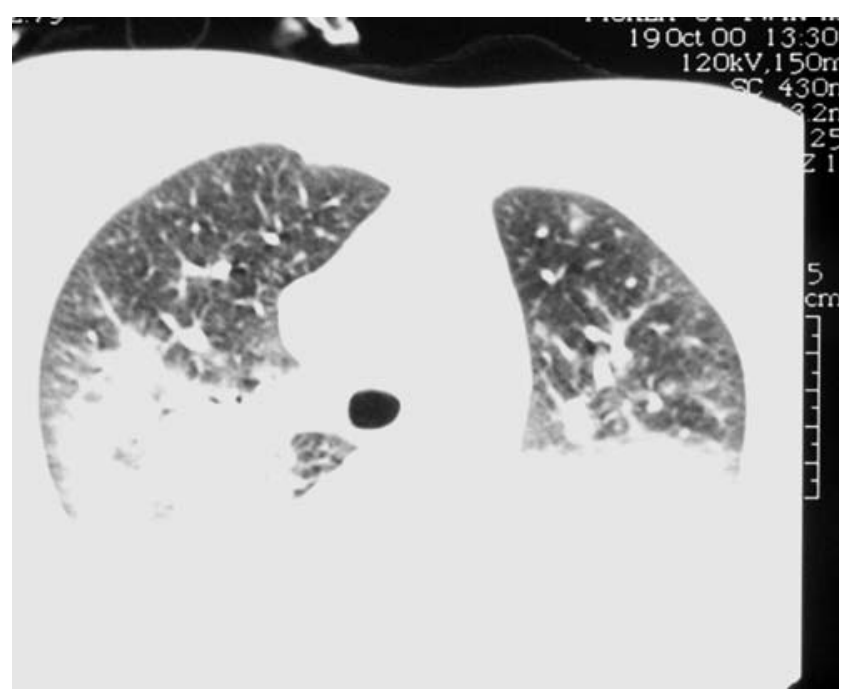

Abb. 5 CT-Thorax kurz nach Aufnahme. Darstellung des Lungenparenchyms. Bds. ausgeprägte Infiltrationen dorsobasal

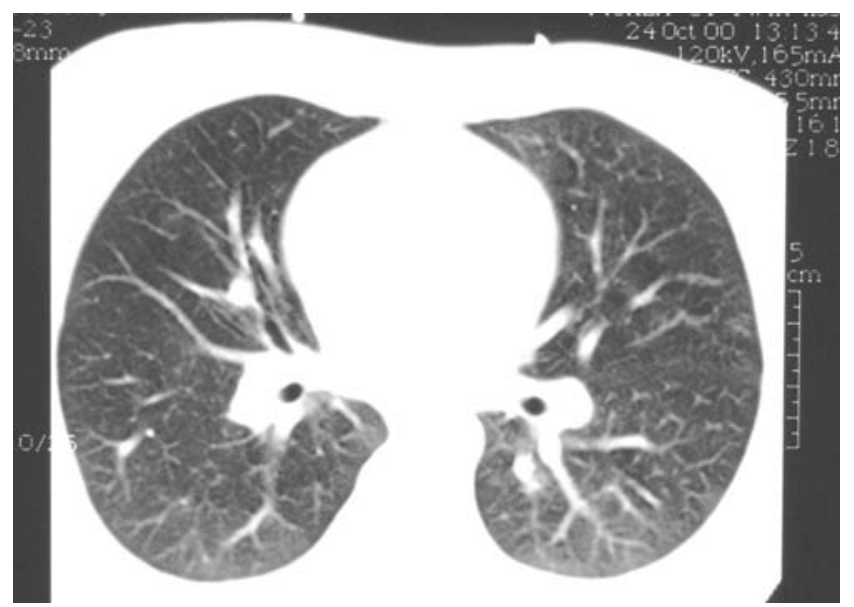

Abb. 6 Kontroll-CT-Thorax vor Verlegung. Darstellung des Lungenparenchyms. Vollständige Rückbildung der Infiltrate

der Organperfusion eine großzügige Volumensubstitution mit einer Einfuhr von 3-4l kristalloider Lösungen pro Tag mit dem Ziel eines zentralvenösen Druckes von $10 \mathrm{mmHg}$. Begleitend erfolgte eine lowdose Heparinisierung. Bei initial noch unbekanntem Erreger leiteten wir eine breite antibiotische Kombinationstherapie mit Amoxicillin, Clavulansäure und Ciprofloxacin ein. Zur Sekretolyse und als ,Radikalenfänger' führten wir eine Therapie mit Acethylcystein durch. Eine Steroidapplikation wurde zwar diskutiert, erfolgte aber nicht. Physikalisch wurde auf eine intensive zweistündliche Lagerungstherapie mit wechselseitiger $120^{\circ}$ Seitenlagerung geachtet [23-25], was aufgrund der CPAP-Maske einer überdurchschnittlichen Zuwendung durch das Pfle- gepersonal bedurfte. Unter den genannten therapeutischen Maßnahmen, CPAP-Atmung mit 100\% Sauerstoff und einem positiven endexspiratorischen Druck von $5 \mathrm{mmHg}$ konnte ein Anstieg des $\mathrm{PaO}_{2}$ von 33 auf $50 \mathrm{mmHg}$ erreicht werden. Hierunter ließ sich die Patientin intensivmedizinisch führen. Im Verlauf waren $a b$ dem zweiten intensivmedizinischen Therapietag rückläufige Entzündungsparameter, eine Normalisierung der Körpertemperatur und eine Besserung der kardiozirkulatorischen Situation zu verzeichnen. Unter Fortführung der nichtinvasiven CPAP-Atmung stabilisierte sich die Respiration $\mathrm{zu}$ nehmend, am dritten Beatmungstag konnte der $\mathrm{FiO}_{2}$ auf 0,6 gesenkt werden. Es erfolgte weiterhin eine konsequente physikalische Atemwegstherapie. Bei einem $\mathrm{PaO}_{2}$ von $94 \mathrm{mmHg}$ wurde am vierten Tag das Beatmungsregime auf intermittierende CPAP-Atmung gelockert. Blutbild, Gerinnungsparameter und CRP normalisierten sich im Verlauf. Eine RöntgenThorax-Verlaufskontrolle am 6. Behandlungstag zeigte eine komplette Rückbildung der initial ausgedehnten Infiltrate (Abb. 2). Auch die CT-Kontrolle der Lunge ergab eine Rückbildung der ausgedehnten Exsudationen, mit nur noch geringer Resteintrübung der zentralen Lungenpartien und der Unterlappen (Abb. 4, 6). Zur Vermeidung eines Rückfalls wurde die Patientin unter intensiver Atemgymnastik und intermittierender CPAP-Atmung noch weitere fünf Tage intensivmedizinisch geführt. Nach Entlassung in die pulmonologische Anschlussheilbehandlung (AHB) erfolgte im Verlauf eine Entfernung des Doppel-J-Katheters und eine extrakorporale Lithotripsie des Konkrements. Dem AHB-Bericht war eine komplette pulmonale Restitutio mit unauffälliger Spirometrie zu entnehmen.

\section{Diskussion}

Bei der hier vorgestellten Pat. kam es im Zusammenhang mit einer Urosepsis zur Entwicklung einer akuten respiratorischen Insuffizienz mit einem $\mathrm{PaO}_{2} /$ $\mathrm{FiO}_{2}$-Quotienten von ca. $82 \mathrm{mmHg}$, beidseitigen, diffusen Lungeninfiltrationen bei echokardiographisch unauffälligem linken Herzen, so dass alle Kriterien zur Diagnose eines ARDS entsprechend der Amerikanisch-Europäischen Konsensus-Konferenz erfüllt waren (Tab. 1). Da die Patientin die Atemnot relativ gut tolerierte und sich als sehr kooperativ erwies, sich in der BGA bei ausgeglichenem Säure-BasenHaushalt noch kein Anhalt für eine vermehrte Hypoxie-bedingte Laktatproduktion zeigte, versuchten wir zunächst auf eine invasive Beatmung zu verzichten. Die Gründe, die für ein nichtinvasives Vorgehen 
auch bei dem ARDS sprechen könnten, möchten wir nachfolgend kurz erläutern.

Es gilt heute als gesichert, dass die maschinelle Beatmung selbst zu einer Schädigung des Lungengewebes mit Verschlechterung des Gasaustausches bis hin zum ARDS, führen kann $(9,26]$. Welche Mechanismen dieser Schädigung zugrunde liegen, ist bisher noch nicht endgültig geklärt. Pathophysiologische Überlegungen und Experimente führten zu dem Konzept des Volu- bzw. Barotraumas. Hierbei kommt es durch die Überdruckbeatmung mit steigenden Tidalvolumina bzw. Beatmungsdrücken zu zunehmenden Scherkräften, die die Elastizität des Lungengewebes überfordern können. Besonders bei dem für das ARDS typischen Nebeneinander gesunder und schwerst veränderter, nicht belüfteter Lungenabschnitte, könnten so schon bei „normalen“ Tidalvolumina erhebliche Scherkräfte einwirken [27]. Folgerichtig wurde für das ARDS die sogenannte „lungenprotektive" Beatmung entwickelt, mit kleinen Atemzugvolumina und resultierend niedriger Beatmungsspitzendrücke unter Tolerierung steigender arterieller $\mathrm{CO}_{2}$-Konzentrationen [28]. Der klinische Vorteil dieser Beatmungsstrategie konnte inzwischen eindrucksvoll belegt werden [9].

Noch einen Schritt weiter geht das Konzept, auf eine invasive Beatmung mit endotrachealer Intubation zugunsten einer Maskenbeatmung zu verzichten, soweit dies die Beatmungsparameter zulassen.

Die Vorteile der nichtinvasiven Beatmung sind das fehlende Intubationstrauma, erhaltene Schutzreflexe, weniger nosokomiale Pneumonien [29, 30], erhaltene Darmmotilität mit geringerem Risiko einer bakteriellen Translokation und geringerer Muskelabbau. Da der Patient einen Teil der Atemarbeit selbst leistet, können die applizierten positiven Atemwegsdrücke deutlich reduziert werden, mit der Folge geringerer zirkulatorischer und renaler Nebenwirkungen sowie eines reduzierten Risikos der Entwicklung eines Volu- bzw. Barotraumas. Die erhaltene Zwerchfellatmung wirkt zudem der Atelektasenbildung in den abhängigen Lungenpartien entgegen, das Rechts-Links-Shuntvolumen sinkt [31]. Letzteres gleicht zumindest teilweise den Nachteil der Maskenbeatmung aus, im Vergleich zur Intubationsbeatmung nur unvollständig einen positiven endexspiratorischen Druck zur Vermeidung des Kollabierens der Alveolen gewährleisten zu können. Schließlich wird die Intubation selbst als Ursache beatmungsinduzierter Lungenschädigungen angesehen, da der Tubus durch ein verändertes Keimspektrum im oropharyngealen Raum zu absteigenden nosokomialen Pneumonien prädestinieren soll $[13,14]$.

Als bisher Einziger publizierte Antonelli 1998 eine randomisierte Vergleichsstudie bei 64 Pat. mit ALI (acute lung injury) oder ARDS, die entweder invasiv oder nicht-invasiv beatmet wurden [13]. Bei knapp $70 \%$ der nicht-invasiv beatmeten konnte eine Intubation vermieden werden. Zwar wurde die Letalität nicht signifikant beeinflusst, doch traten in der nicht-invasiv beatmeten Gruppe sekundäre Organkomplikationen signifikant seltener auf (38\% vs. $66 \%)$. Besonders eklatant war der Unterschied bei nosokomialen Pneumonien (3\% nicht-invasiv vs. $25 \%$ invasiv). Insgesamt zeigte die nichtinvasive Gruppe signifikant weniger sekundäre Organkomplikationen mit kürzeren Beatmungs- und Verweildauern.

In der septischen Kreislaufdysregulation mit ausgeprägter peripherer Vasodilatation und Abfall des peripher-vaskulären Widerstandes ist ein massiv erhöhtes Herzzeitvolumen zur Aufrechterhaltung des aeroben Metabolismus der nichtpulmonalen Organe erforderlich [32]. Eine volumenrestriktive Therapie bei hyperdynamer Kreislaufsituation verschlechtert die metabolische Situation des Gesamtorganismus durch Reduktion des erforderlichen Herzzeitvolumens. Tuchschmidt et al. analysierten in einer retrospektiven Studie an 78 Patienten im septischen Schock prädiktive Parameter für den Endpunkt Mortalität [33]. Als statistisch signifikant erwiesen sich das Herzzeitvolumen und das Sauerstoffangebot. Häufigste Ursache des ARDS ist jedoch eine Sepsis mit Vorliegen einer hyperdynamen Kreislaufsituation und unter konventioneller Therapie ist gerade beim Sepsis-assoziierten ARDS die Mortalität besonders hoch [34]. Bezüglich der Volumenbilanzierung besteht bei einem Sepsis-bedingten ARDS somit ein „therapeutisches Dilemma“.

In dem hier beschriebenen Fall entschieden wir uns aufgrund der zuvor ausgeführten Überlegungen und der hervorragenden Mitarbeit der Patientin für eine nichtinvasive CPAP-Beatmung und ein liberales Volumenmanagement in Kombination mit regelmäßigem Wechsel zwischen Rücken- und Bauchlagerung. Hierunter beobachteten wir einen erstaunlich positiven Verlauf. Auch wenn die komplette Restitution sicherlich auch Folge des jungen Alters und der fehlenden Komorbidität der Patientin ist, so ermutigt dieser Fall u. E. aber doch auch, beim Sepsis-bedingten ARDS das Konzept der nichtinvasiven Beatmung und der liberalen Volumenbilanzierung häufiger in Erwägung zu ziehen.

\section{Fazit}

Als Therapie der Wahl beim ARDS gilt bis dato die invasiv-maschinelle Beatmung mit niedrigen Tidalvolumina, niedrigen Beatmungsspitzendrücken und permissiver Hyperkapnie, begleitet von einem restriktiven Volumenmanagement. Wir berichten über 
den Fall eines schweren ARDS im Rahmen einer Urosepsis bei einer jungen, bisher gesunden Patientin. Den erfreulich positiven Verlauf mit kompletter
Restitution sehen wir im Zusammenhang mit einem nichtinvasiven Beatmungsregime und liberaler Volumenbilanzierung.

\section{Literatur}

1. Ashbaugh DG, Bigelow DB, Petty TL Levine BE (1967) Acute respiratory distress in adults. Lancet 2:319-323

2. Bernard GR, Artiga A, Brigham KL, Carlet J, Falke K, Hudson L, Lamy M, Legall JR, Morris A, Spragg R (1994) The American-European Consensus Conference on ARDS. Definitions, mechanisms, relevant outcomes and clinical trial coordination. Am J Repir Crit Care Med 149:818-824

3. Walmrath D, Günther A, Grimminger F, Seeger W (2000) Das alveoläre Surfactantsystem: seine pathogenetische Bedeutung für das akute Lungenversagen und therapeutische Perspektiven. Intensivmed 37:307-317

4. Fabel H, Krause J (1995) Akutes Lungenversagen. In: Fabel H. Pneumonologie. Urban und Schwarzenberg, München

5. Hien P (2000) Praktische Pneumonologie. Springer, Berlin

6. Tomashefski JF (1994) Noncardiogenic pulmonary edema and diffuse alveolar damge. In: Saldana MJ. Pathology of Pulmonary Disease. JB Lippincott Company, Philadelphia

7. Gerlach H (2001) Diagnostik des ARDS. Dtsch Med Wochenschr 126: 1139-1143

8. Galanski M, Prokop M (1998) Ganzkörper-Computertomographie. Thieme, Stuttgart, S 172

9. The Acute Respiratory Distress Syndrome Network (2000) Ventilation with lower tidal volumes as compared with traditional tidal volumes for acute lung injury and the acute respiratory distress syndrome. $\mathrm{N}$ Engl J Med 342:1301-1308

10. Jürgens E, Kuhlen R, Max M, Rossaint R (2001) Beatmungsstrategie beim akuten Lungenversagen. Intensivmed 38:601-610

11. Kopp R, Kuhlen R, Max M, Roissant $R$ (2002) Evidence-based medicine in the therapy of the acute respiratory distress syndrome. Intensive Care Med 28:244-255

12. Eisner MD, Thompsen BT, Schoenfeld D, Anzueto A, Matthay MA (2002) Airway pressures and early barotrau$\mathrm{ma}$ in patients with acute lung injury and acute respiratory distress syndrome. Am J Respir Crit Care Med 165:978-982
13. Antonelli M, Conti G, Rocco M, Bufi M, De Blasi RA, Vivino G, Gasparetto A, Meduri GU (1998) A comparison of noninvasive positive pressure ventilation and conventional mechanical ventilation in patients with acute respiratory failure. N Engl J Med 339: 429-435

14. Walmrath D, Grimminger F, Seeger W (2000) Non-Invasive Beatmung des akuten hypoxämischen Lungenversagens - State of the Art 2000. Intensivmed 37:251-256

15. Humphrey H, Hall J, Sznajder I, Siverstein M, Wood L (1990) Improved survival in ARDS patients associated with a reduction in pulmonary capillary wedge pressure. Chest 97:1176-1180

16. Rivers E, Nguyen B, Havstad S, Ressler J, Muzzin A, Knoblich B, Peterson E, Tomlanivich M (2001) Early goaldirected therapy in the treatment of severe sepsis and septic shock. N Engl J Med 345:1368-1377

17. Schuller D, Schuster DP (1997) The rational for fluid restriction during treatment of ARDS. Sem Respir Crit Care Med 18:33-38

18. Dreyfuss D, Saumon G (2002) Evidence-based medicine or fuzzy logic: what is the best for ARDS management? Intensive Care Med 28:230-234

19. Tuchschmidt J, Fried J, Astiz M, Rackow E (1992) Elevation of cardiac output and oxygen delivery improves outcome in septic shock. Chest 102:216-220

20. Tuchschmidt JA, Mecher CE (1994) Predictors of outcome from critical illness, shock and cardiopulmonary resuscitation. Crit Care Clin 10:179 195

21. Walmrath D, Grimmingerr F, Seeger W (1999) Therapie des ARDS. Intensivmed 36:104-125

22. Gerlach H (2001) Therapie des ARDS. DMW 126:1173-1177

23. Ward NS (2002) Effects of prone position ventilation in ARDS. An evidence-based therapyreview of the literature. Crit Care Clin 18(1):35-44

24. Lee DL, Chiang HAT, Lin SL, Ger LP, Kun MH, Huang YC (2002) Prone-Position ventilation induces sustained improvement in oxygenation in patients with acute respiratory distress syndrome who have a large shunt. Crit Care Med 30:1446-1452
25. Pelosi P, Brazzi L, Gattinoni L (2002) Prone position in acute respiratory distress syndrome. Eur Respir J 20: 1017-1028

26. Jürgens E, Roissaint R (2000) Invasive Beatmung beim hypoxämischen Lungenversagen - state of the art. Intensivmed. 37:257-264

27. Mead J, Takishima T, Leith D (1970) Stress distribution in lungs: a model of pulmonary elasticity. J Appl Physiol 28:596-608

28. Hickling KG, Henderson SJ, Jackson $\mathrm{R}$ (1990) Low mortality associated with low volume pressure limited ventilation with permissive hypercapnia in severe adult respiratory distress syndrome. Intenive Care Med 16:372-377

29. Estes RJ, Meduri GU (1995) The pathogenesis of ventilator-associated pneumonia. I. Mechanisms of bacterial trancolonization and airway inoculation. Intensive Care Med 21: 363-383

30. Guerin C, Girard R, Chemorin C, De Varax R, Fournier G (1997) Facial mask noninvasive mechanical ventilation reduces the incidence of nosocomial pneumonia: a prospective epidemiological survey from a single ICU. Intensive Care Med 23:1024-1032

31. Sydow M, Burchardi H, Ephraim E, Zielmann S, Crozier T (1994) Longterm effects of two different ventilatory modes on oxygenation in acute lung injury. Comparison of airway pressure release ventilation and volume controlled inversed ratio ventilation. Al J Respir Crit Care 149: 15501556

32. Tuchschmidt J, Oblitas D, Fried JC (1991) Oxygen consumption in sepsis and septic shock. Crit Care Med 19(5):664-671

33. Tuchschmidt J, Fried J, Swinney R, Sharma OP (1989) Early haemodynamic correlates of survival in patients with septic shock. Crit Care Med 17(8):719-723

34. Fabel H, Krause J (1995) Akutes Lungenversagen. In: Fabel H. Pneumonologie; Urban und Schwarzenberg, München 